\title{
The emerging role of ferroptosis in intestinal disease
}

\author{
Shu Xu', Yao He', Lihui Lin', Peng Chen', Minhu Chen and Shenghong Zhang ${ }^{1}$
}

\begin{abstract}
Ferroptosis is a newly recognised type of regulated cell death (RCD) characterised by iron-dependent accumulation of lipid peroxidation. It is significantly distinct from other RCDs at the morphological, biochemical, and genetic levels. Recent reports have implicated ferroptosis in multiple diseases, including neurological disorders, kidney injury, liver diseases, and cancer. Ferroptotic cell death has also been associated with dysfunction of the intestinal epithelium, which contributes to several intestinal diseases. Research on ferroptosis may provide a new understanding of intestinal disease pathogenesis that benefits clinical treatment. In this review, we provide an overview of ferroptosis and its underlying mechanisms, then describe its emerging role in intestinal diseases, including intestinal ischaemia/ reperfusion (I/R) injury, inflammatory bowel disease (IBD), and colorectal cancer (CRC).
\end{abstract}

\section{Facts}

- Ferroptosis is a unique type of regulated cell death that involves iron accumulation and lipid oxidation.

- Ferroptosis has been linked to several diseases and cancers, but its role in intestinal disease is uncharacterised.

- Ferroptosis can be a positive and negative regulator of the disease.

\section{Open questions}

- Does ferroptosis play a role in distinct forms of intestinal diseases?

- What contributes to ferroptosis in the occurrence and development of intestinal diseases? Are there unknown mechanisms and signalling pathways?

- Will ferroptosis-related factors be indicators of disease severity?

Correspondence: Minhu Chen (chenminhu@mail.sysu.edu.cn) or

Shenghong Zhang (zhshh3@mail.sysu.edu.cn)

'Division of Gastroenterology, The First Affiliated Hospital, Sun Yat-sen

University, Guangzhou, P. R. China

These authors contributed equally: Shu Xu, Yao He

Edited by F. Pentimall

\section{Introduction}

Ferroptosis is a form of regulated cell death (RCD) that was first proposed by Dixon and colleagues in $2012^{1}$. It is morphologically, biochemically, and genetically different from other kinds of RCD, such as apoptosis, necroptosis, and autophagy ${ }^{1,2}$. Iron metabolism and the lipid peroxidation pathway are central mediators of the ferroptotic process $^{3,4}$ (Fig. 1). Excessive iron regulates ferroptosis by producing lethal reactive oxygen species (ROS) via the Fenton reaction, while reduced glutathione (GSH) depletion and/or glutathione peroxidase 4 (GPX4) inhibition trigger ferroptosis through the accumulation of intracellular lipid ROS and overwhelming lipid peroxidation ${ }^{1,4,5}$. In addition, ROS attack the polyunsaturated fatty acids (PUFAs) of lipid membranes, producing massive lipid peroxides and leading to membrane damage and cell death $^{4,6}$. Specific small-molecule compounds, such as erastin and RAS-selective lethal 3 (RSL3) can induce ferroptosis, while ferrostatin-1 (Fer-1), liproxstatin-1 (Lip-1), and iron chelators deferoxamine (DFO) inhibit $\mathrm{it}^{7,8}$. Accumulating evidence suggests that ferroptosis participates in multiple diseases, including neurological disorders, ischaemia/reperfusion (I/R) injury, kidney failure, cardiac disease, and cancer ${ }^{1,4,9-11}$. Recent studies have also implicated ferroptosis in intestinal diseases, including

\section{(c) The Author(s) 2021}

(c) Open Access This article is licensed under a Creative Commons Attribution 4.0 International License, which permits use, sharing, adaptation, distribution and reproduction cc) in any medium or format, as long as you give appropriate credit to the original author(s) and the source, provide a link to the Creative Commons license, and indicate if changes were made. The images or other third party material in this article are included in the article's Creative Commons license, unless indicated otherwise in a credit line to the material. If material is not included in the article's Creative Commons license and your intended use is not permitted by statutory regulation or exceeds the permitted use, you will need to obtain permission directly from the copyright holder. To view a copy of this license, visit http://creativecommons.org/licenses/by/4.0/. 


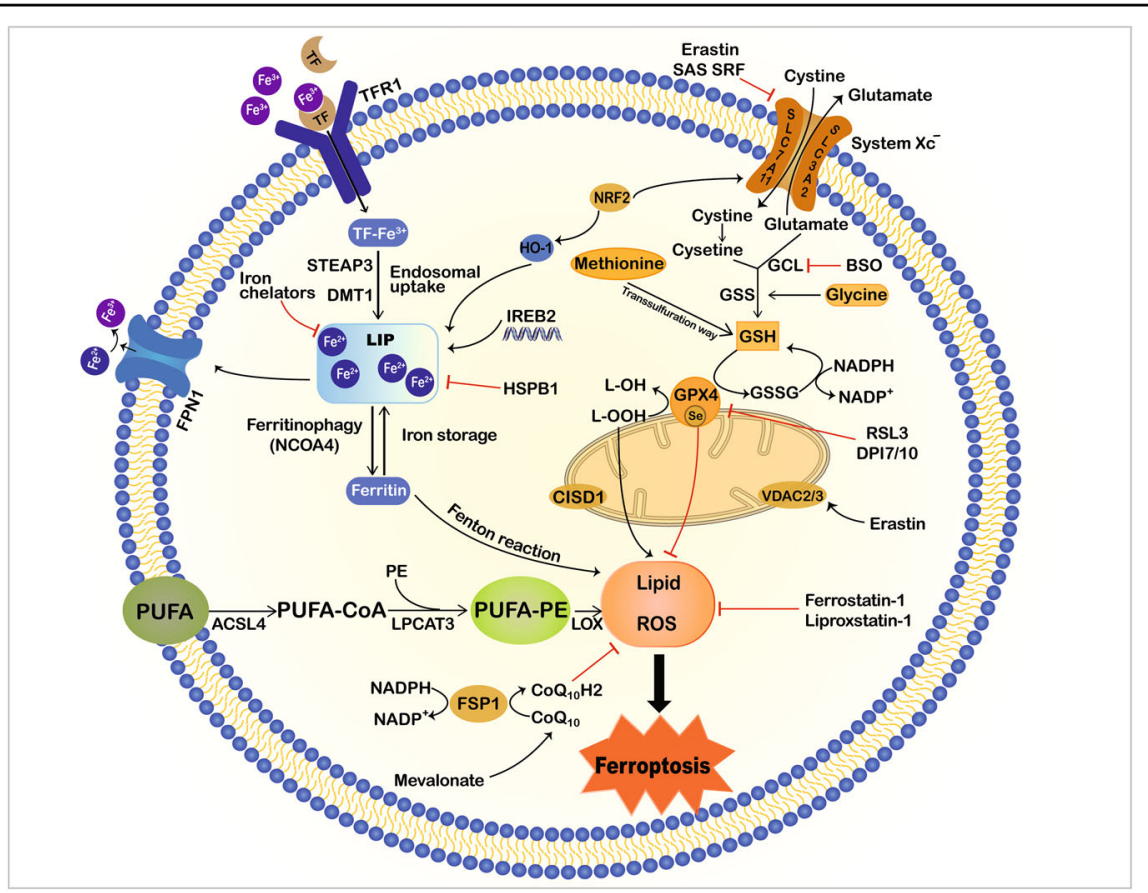

Fig. 1 Mechanisms of ferroptosis. Ferroptosis is characterised by iron accumulation, excessive ROS production and overwhelming lipid peroxidation. Three main metabolic pathways, amino-acid/GSH, lipid, and iron pathways, participate in the initiation and execution of ferroptosis. Moreover, there are additional signalling pathways and regulators controlling ferroptosis sensitivity. This illustration shows the process of ferroptosis, summarising the key molecules and targets that regulate iron and lipid peroxidation. ACSL4 acyl-COA synthetase long-chain family member 4, BSO buthionine sulphoximine, CISD1 CDGSH iron sulphur domain 1, DMT1 divalent metal transporter 1, FSP1 ferroptosis suppressor protein 1, FPN1 ferroportin 1, GPX4 glutathione peroxidase 4, GSH glutathione, GSSG oxidized glutathione, GSS glutathione synthetase, GCL glutamate-cysteine ligase, HO-1 haem oxygenase 1, HSPB1 heat shock protein beta-1, IREB2 iron response element-binding protein 2, LOX lipoxygenase, LPCAT3 lysophosphatidylcholine acyltransferase 3, NCOA4 nuclear receptor coactivator 4, NRF2 nuclear factor E2-related factor 2, PUFA polyunsaturated fatty acid, PE phosphatidylethanolamine, ROS reactive oxygen species, RSL3 Ras-selective lethal 3, STEAP3 six-transmembrane epithelial antigen of prostate 3 metalloreductase, SLC7A11 solute carrier family 7 member 11, SAS sulphasalazine, SRF sorafenib, TF transferrin, TFR1 transferrin receptor 1, VDAC2/3 voltage dependent-anion channel 2/3.

intestinal I/R injury, inflammatory bowel disease (IBD), and colorectal cancer (CRC) $)^{12-16}$ (Fig. 2 and Table 1). Ferroptosis has been reported in ulcerative colitis (UC) in both humans and mice; moreover, blocking the ferroptotic process alleviated dextran sulphate sodium (DSS)-induced colitis $^{12,13}$. Furthermore, ferroptosis can limit the migration, invasion, and proliferation of CRC. Indeed, RSL3 drives ferroptotic cell death by promoting cellular ROS accumulation and increasing iron load ${ }^{17}$. Another report indicated that in CRC, resibufogenin inhibited cell growth and tumorigenesis by inducing ferroptosis through GPX4 inactivation ${ }^{18}$. Taken together, ferroptosis appears to play a key role in the pathophysiological processes and may provide new ideas and means for the treatment of intestinal diseases. This review presents a comprehensive description of ferroptosis and its emerging role in multiple intestinal diseases.

\section{Ferroptosis: an iron-dependent type of regulated cell death with clinical significance Definition and measurement}

Since 2003, Stockwell and colleagues have successively identified novel compounds, including erastin and RSL3, that activate new, nonapoptotic cell death in particular cancer cells ${ }^{19,20}$. Inhibitors specific to known RCDs did not inhibit this chemically induced cell death; however, antioxidants and iron chelators could block and reverse the process $^{21}$. The definition of ferroptosis was proposed in 2012: nonapoptotic, iron-dependent cell death characterised by the accumulation of lipid peroxidation products and the depletion of membrane PUFAs ${ }^{1}$. Ferroptosis was added to the RCD family by the Nomenclature Committee on Cell Death (NCCD) in $2018^{22}$. Morphologically, ferroptosis manifests as small mitochondria with concentrated membrane density, decreased or vanishing mitochondrial cristae, and outer mitochondrial membrane rupture ${ }^{4,23}$. The biochemical properties of ferroptosis are iron accumulation, lethal ROS production, and overwhelming lipid peroxidation ${ }^{4,10}$. Multiple molecules, including GPX4, p53, solute carrier family 7 member 11 (SLC7A11), acyl-CoA synthetase long-chain family member 4 (ACSL4), NADPH oxidase (NOX), and nuclear factor E2-related factor 2 (NRF2) positively or negatively regulate ferroptosis ${ }^{1,24-26}$.

To assess ferroptosis, the Cell Counting Kit- 8 and propidium iodide staining can be used to measure cell 


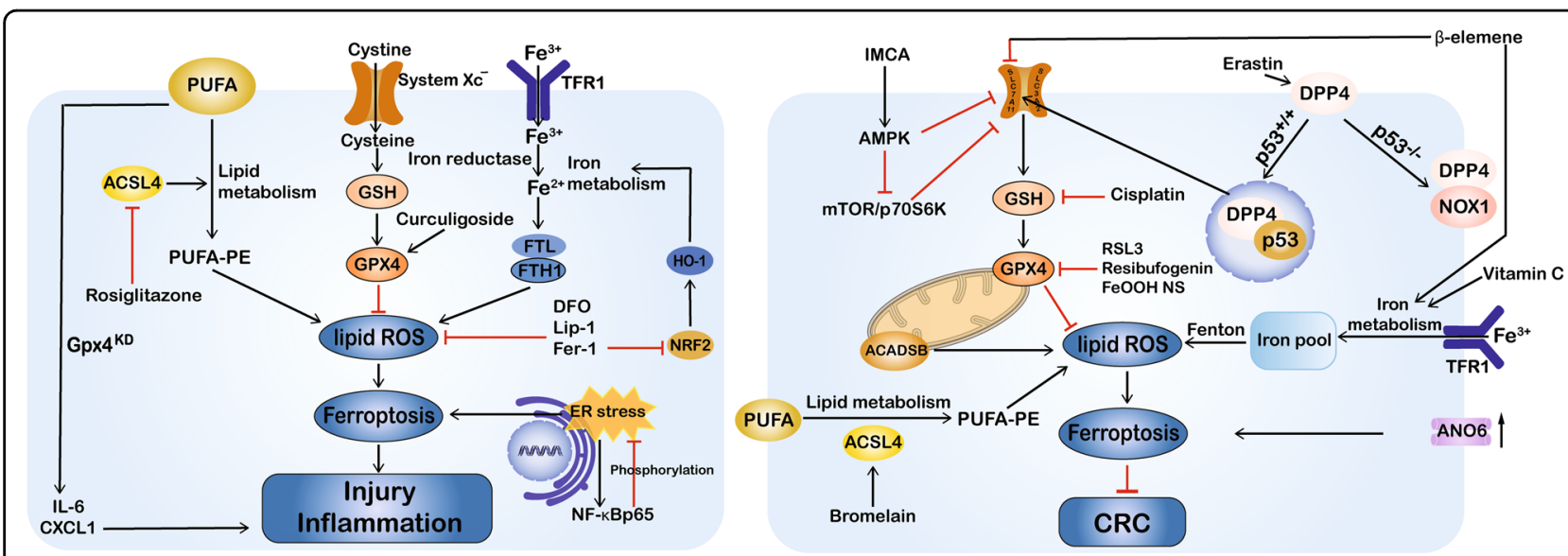

Fig. 2 Dual regulatory roles for ferroptosis in intestinal diseases. Ferroptosis can be a positive and negative regulator of intestinal diseases according to cell type and disease context. The induction of ferroptosis by multiple compounds can inhibit cancer growth; however, inhibiting ferroptosis has an anti-inflammatory effect and can attenuate intestinal injury in IBD and I/R injury. This schematic diagram shows ferroptosis regulators and pathways. ACADSB acyl-Coenzyme A dehydrogenase short/branched chain, ACSL4 acyl-CoA synthetase long-chain family member 4, ANO6 anoctamin 6, CRC colorectal cancer, CXCL1 chemokine (C-X-C motif) ligand 1, DFO deferoxamine, DPP4 dipeptidyl-peptidase-4, ER endoplasmic reticulum, Fer-1 ferrostatin-1, FTL ferritin light chain, FTH1 ferritin heavy chain 1, FeOOH NS iron oxide-hydroxide nanospindle, GPX4 glutathione peroxidase 4, GSH glutathione, HO-1 haem oxygenase 1, IMCA 2-Imino-6-methoxy-2H-chromene-3-carbothioamide, I/R ischaemia/ reperfusion, IBD inflammatory bowel disease, IL-6 interleukin 6, KD knockdown, Lip-1 liproxstatin-1, NOX1 NADPH oxidase 1, NRF2 nuclear factor E2-related factor 2, PUFA polyunsaturated fatty acid, PE phosphatidylethanolamine, ROS reactive oxygen species, RSL3 Ras-selective lethal small molecule 3, SLC7A11 solute carrier family 7 member 11, TFR1 transferrin receptor 1 .

viability and death 9,27 . Measuring lipid peroxidation is important for evaluating the presence of ferroptosis in specific contexts. Oxidative lipidomics is the gold standard for identifying specific oxidized lipids ${ }^{28,29}$. Probes such as C11-BODIPY and Liperfluo provide indirect but efficient means to detect lipid $\operatorname{ROS}^{2,30}$. Moreover, malondialdehyde (MDA) and 4-hydroxynonenal (4-HNE) are common by-products of lipid peroxidation during oxidative stress that allow the measurement of lipid peroxidation $^{31}$. Another method for evaluating ferroptosis is to test cellular iron levels using an iron assay kit or the fluorescent probe Phen Green SK (PGSK) ${ }^{20,32}$. We can also detect changes in ferroptosis-related gene expression, such as prostaglandin-endoperoxide synthase (PTGS), ACSL4, GPX4, and ferritin heavy chain $1(F T H 1)^{33}$. In addition, transmission electron microscopy can be used to identify specific morphological features of cells to support ferroptosis occurrence ${ }^{34}$.

\section{Mechanisms and mediators of ferroptosis GSH/GPX4-lipid peroxidation pathway}

Ferroptosis is triggered by excessive lipid peroxidation arising from iron-dependent ROS accumulation. As it can occur when GSH-dependent lipid peroxide repair systems are compromised, maintaining ROS and lipid peroxides at physiological concentrations is a critical factor in minimizing susceptibility ${ }^{2,35}$. Lipophilic antioxidants (e.g. Fer-1, Lip-1) have been defined as specific ferroptosis suppressors that inhibit ROS accumulation caused during lipid oxidation. GSH is a thiol-containing tripeptide that plays an essential role in intracellular antioxidant defences. Its depletion causes increased oxidative stress, macromolecular damage, and subsequent cell death ${ }^{36}$. GPX4 is a member of the glutathione peroxidase family capable of reducing cytotoxic lipid hydroperoxides (L-OOH) to non-toxic lipid alcohols $(\mathrm{L}-\mathrm{OH})$ or catalysing free hydrogen peroxide into water to prevent the formation and accumulation of lethal lipid ROS at the expense of $\mathrm{GSH}^{37,38}$.

Accumulating evidence has implicated GPX4 as a master regulator of ferroptosis; its inhibition by pharmacological or genetic methods can trigger ferroptotic cell death through the accumulation of lipid peroxides ${ }^{5,39,40}$. Indeed, RSL3 has been shown to induce ferroptosis by directly inhibiting GPX4 activity through covalent binding with the selenocysteine active site of GPX $4^{5,41}$. GPX4 can also be inactivated by indirect methods, such as cellular GSH depletion. The biosynthesis of GSH requires the participation of glutamate, cysteine, and glycine in a twostep reaction catalysed by glutamate-cysteine ligase (GCL) and glutathione synthetase (GSS) ${ }^{42,43}$. Thus, GSH depletion can result either from direct inhibition of GSH synthesis (e.g. by the known GCL inhibitor buthionine sulphoximine $(\mathrm{BSO})^{6}$ ) or from cysteine/glutamate unavailability. Cysteine, the rate-limiting substrate for GSH biosynthesis, is produced from dipeptide cystine imported by the cell surface cystine/glutamate antiporter system $\mathrm{X}_{\mathrm{c}}{ }^{-}$, or from methionine via the transsulphuration 


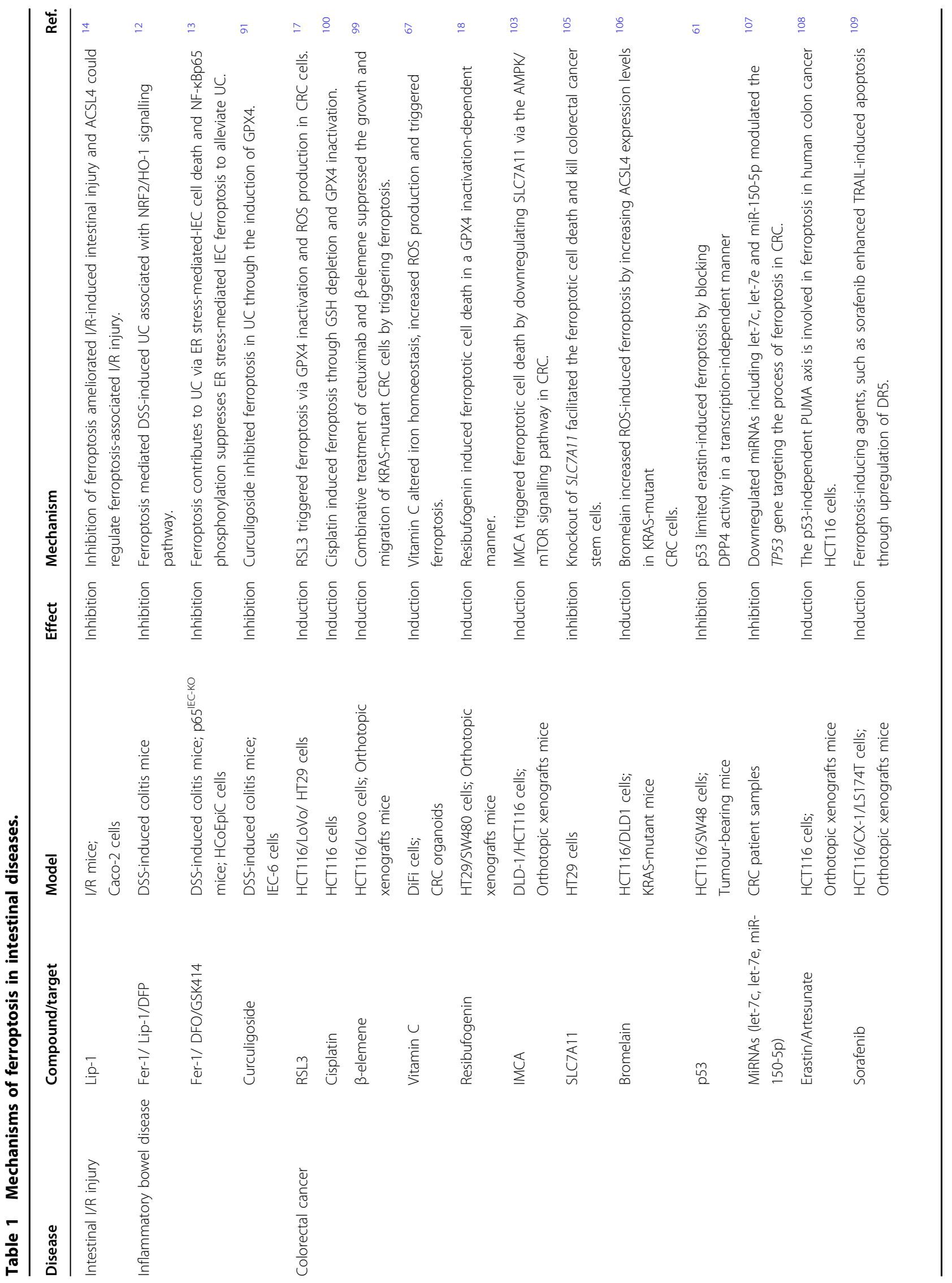




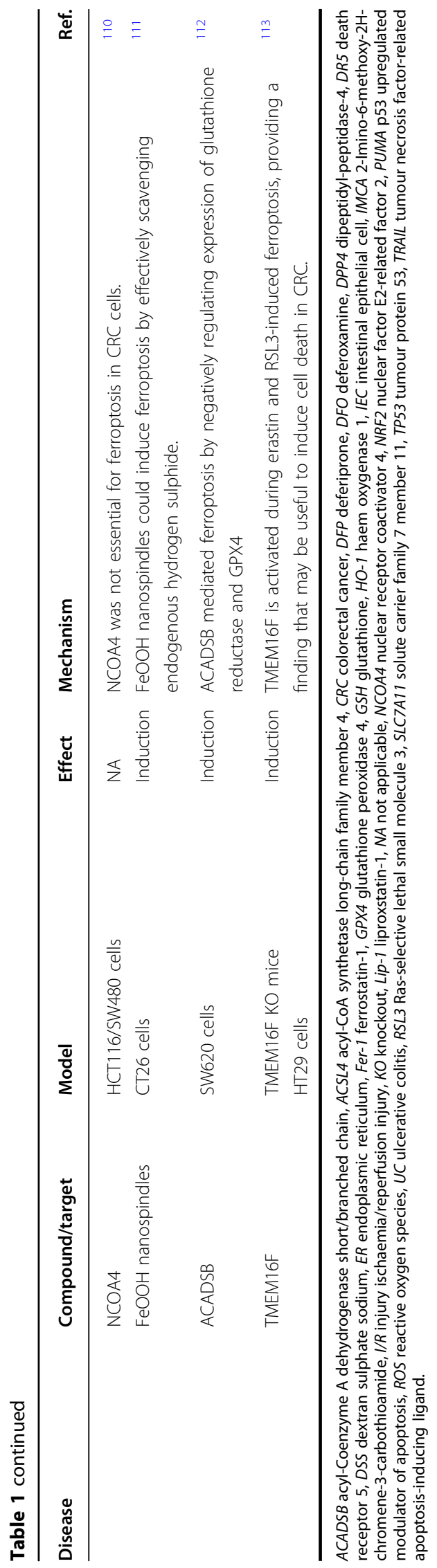

pathway ${ }^{44,45}$. Inhibiting system $\mathrm{X}_{\mathrm{c}}{ }^{-}$can reduce $\mathrm{GSH}$ levels and GPX4 activity, contributing to ferroptotic cell death. Erastin and other molecules (e.g. sulphasalazine, sorafenib) are inhibitors of system $\mathrm{X}_{\mathrm{c}}^{-}$and thus induce ferroptosis ${ }^{1,3,4}$. Studies have shown that regulating the expression of SLC7A11, the functional subunit of system $\mathrm{X}_{\mathrm{c}}{ }^{-}$, affects system $\mathrm{X}_{\mathrm{c}}{ }^{-}$activity and ferroptosis sensitivity in cancer cells ${ }^{24,26,44}$. Furthermore, cysteinyl-tRNA synthetase (CARS) has been found to participate in the transsulphuration pathway; its knockdown causes upregulation of this pathway and erastin-induced ferroptosis resistance ${ }^{2}$.

As described below, disruption of lipid repair systems involving GSH and GPX4 can facilitate the accumulation of (lipid) ROS; however, cysteine/GSH depletion and/or GPX4 suppression alone is not sufficient to cause ferroptosis. ROS react with PUFAs of lipid membranes to cause lipid peroxidation, which is central to the final execution of ferroptosis. Free PUFAs are substrates for the synthetic-lipid signal-transduction medium, but they must be esterified and incorporated into membrane phospholipids (PLs) with the help of the enzymes ACSL4 and lysophosphatidylcholine acyltransferase 3 (LPCAT3). Then lipoxygenases (LOXs) catalyse PUFA-containing PLs to produce pro-ferroptotic lipid peroxidation ${ }^{23}$. Researchers have identified ACSL4 as both a biomarker for, and a critical contributor to, ferroptosis ${ }^{25,46}$. ACSL4 expression is positively correlated with ferroptosis sensitivity; in addition, lipid oxidation upon GPX4 inhibition requires the involvement of ACSL4 ${ }^{25,46}$. One group has reported that LPCAT3 deletion protected fibroblasts against ferroptosis, suggesting that LPCAT3 is also an important player in ferroptosis ${ }^{25}$. However, this protective effect was mild compared with the protection provided by ACSL4 deletion, suggesting that ACSL4 plays a more extensive role in ferroptosis; moreover, the functional effect of LPCAT3 possibly depends on cellular subtypes $^{25,47}$. Of the different oxidised PL species, PUFAcontaining phosphatidylethanolamines (PEs), especially arachidonic acid (AA)- and adrenic acid (AdA)-containing PEs, are the most susceptible to peroxidation in ferroptosis $^{48}$. Finally, overwhelming lipid peroxidation likely alters lipid bilayer properties, producing cytotoxic reactive fragments, and leading to irreversible cell death ${ }^{49}$.

\section{Iron metabolism and ferroptosis}

Iron is a redox-active element that promotes ROS generation through the Fenton reaction, which leads to non-enzymatic lipid peroxidation ${ }^{3,50}$. Iron also serves as a cofactor for iron-containing enzymes, including LOXs, suggesting a necessary role in enzymatic lipid reactions ${ }^{50}$. Thus, as a significant factor for the production of (lipid) ROS via enzymatic or non-enzymatic ways, iron appears to be an indispensable component in ferroptosis ${ }^{1,2}$. 
The chelation of intracellular iron by DFO and ciclopirox olamine is sufficient to inhibit erastin-induced cell death, reinforcing the importance of iron in ferroptosis ${ }^{4}$. Normally, extracellular iron forms a complex with circulating transferrin (TF), binds to the specific membrane transferrin receptor protein-1 (TFR1), and is delivered into cells. Excess cellular iron is stored as ferritin, the main intracellular iron storage protein that consists of a ferritin light chain (FTL) polymer and FTH1, or exported by iron exporter ferroportin (FPN $)^{51,52}$. Maintaining cellular iron homoeostasis can prevent oxidative damage, and cell toxicity and death. Either reduced iron storage or increased iron uptake can cause iron overload and trigger ferroptosis ${ }^{4}$. Recent studies have revealed an association between genes involved in iron metabolism and ferroptosis. Erastin-induced ferroptosis can be prevented by silencing TFRC, the gene that encodes TFR1, whereas overexpression of haem oxygenase 1 (HO-1) alters iron homoeostasis and aggravates it ${ }^{6,53}$. Autophagic degradation of ferritin, known as ferritinophagy, modulated by the nuclear receptor coactivator 4 (NCOA4), controls cellular liable iron levels and ROS accumulation, thus regulating ferroptotic cell death in some cell lines ${ }^{54,55}$. The pentaspan membrane protein prominin-2 can drive ferroptosis resistance by promoting the formation of ferritincontaining multivesicular bodies and exosomes, thus exporting iron from the cell ${ }^{56}$. Furthermore, iron response element-binding protein 2 (IREB2) encodes the master regulator of iron metabolism, which results in the expression of TRFC, FTH1, and FTL. Inhibiting IREB2 expression contributes to decreased sensitivity to erastininduced ferroptosis ${ }^{1}$. Other targets, such as heat shock protein beta-1 (HSPB1) and CDGSH iron sulphur domain 1 (CISD1), can also regulate ferroptotic cell death by mediating iron uptake and lipid peroxidation ${ }^{57,58}$. Collectively, these findings indicate the iron dependence of ferroptosis.

\section{Other ferroptosis regulatory pathways}

The canonical tumour suppressor p53 probably plays dual roles in mediating ferroptosis in multiple cancers (Fig. 3). It can directly adjust the metabolic versatility of cells by modulating metabolic targets, favouring mitochondrial respiration and resulting in ROS-mediated cell death $^{59}$. Researchers have found that p53 represses SLC7A11 protein expression, resulting in decreased cystine import, decreased GSH production, and enhanced ROS-mediated ferroptosis in some cancer cell lines ${ }^{24,59}$. The acetylation-defective mutant $\mathrm{p} 53^{3 \mathrm{KR}}$, which lacks the ability to trigger apoptosis, cell-cycle arrest, and senescence, can suppress tumourigenesis by inhibiting SLC7A11 and inducing ferroptosis ${ }^{24}$. On the contrary, other studies have reported an inhibitory effect of p53 on ferroptosis in different contexts. Wild-type p53 stabilisation suppresses

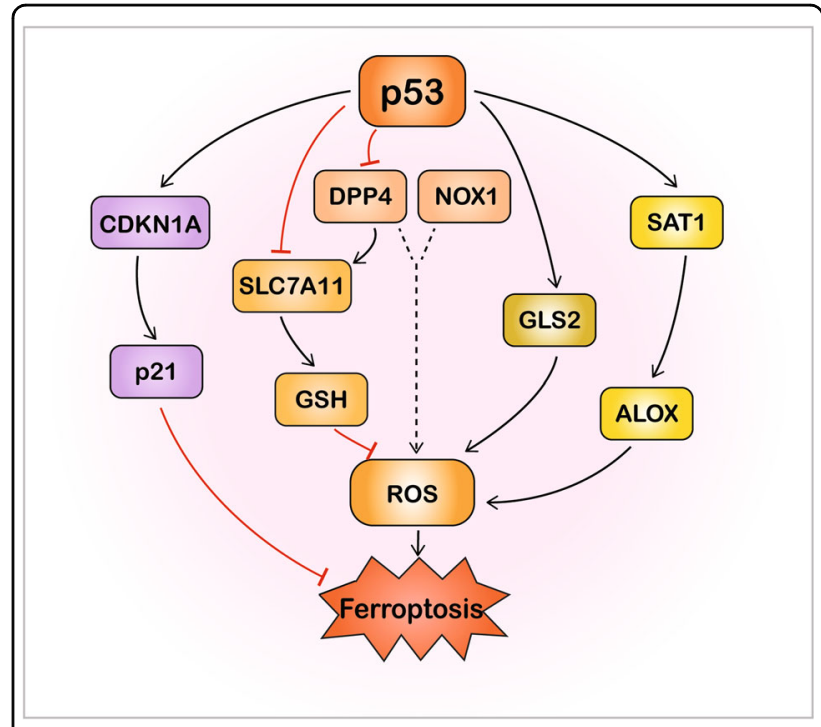

Fig. 3 p53-mediated ferroptosis. p53 plays a dual role in the regulation of ferroptosis through transcriptional or posttranslational mechanisms in different contexts. On one hand, p53 induces ferroptosis through inhibition of SLC7A11 or upregulation of GSL2 and SAT1-ALOX pathway. On the other hand, p53 also inhibits ferroptosis through inhibition of DPP4 activity or by the transcriptional activation of CDKN1A/p21. ALOX arachidonate lipoxygenase, CDKN1A cyclindependent kinase inhibitor 1 A, DPP4 dipeptidyl-peptidase-4, GLS2 glutaminase 2, GSH glutathione, NOX1 NADPH oxidase 1, ROS reactive oxygen species, SAT1 spermidine/spermine N1-acetyltransferase 1, SLC7A11 solute carrier family 7 member 11 .

ferroptosis in specific cancer cell lines in response to cystine deprivation and system $\mathrm{X}_{\mathrm{c}}{ }^{-}$inhibition because of the activation of p53-p21 transcriptional axis ${ }^{60}$. Besides, p53 negatively regulates ferroptosis in CRC cells by inhibiting dipeptidyl-peptidase-4, described in more detail in section 'Ferroptosis and colorectal cancer' below ${ }^{61}$. In addition to p53-mediated ferroptosis, the intracellular metabolic process glutaminolysis is required for the initiation of cystine deprivation-induced ferroptosis ${ }^{9}$. The FSP1-CoQ10-NAD(P)H pathway exists as an independent parallel system that cooperates with GSH/GPX4 to mitigate lipid peroxidation and ferroptosis ${ }^{62}$. Targeting the NRF2-related pathway is also a vital strategy to mediate lipid peroxidation and ferroptosis ${ }^{63}$.

\section{The significance of ferroptosis research in disease}

In parallel with more basic research, it has been found that inducing or blocking ferroptosis can affect the onset and development of multiple pathogenic conditions, providing a potential target for therapeutics, especially for diseases tolerant/resistant to conventional drugs. Taking drug-resistant cancer as an example, ineffective induction of cancer cell death is an important problem with many chemotherapy and bio-targeted drugs, closely linked to drug resistance ${ }^{64}$. Persister cells are clones that survive 
initial cancer treatment and induce drug-resistant states across diverse cancer contexts ${ }^{64,65}$. Interestingly, induction of ferroptosis can kill these drug-tolerant persister cells and decrease the emergence of acquired drug resistance ${ }^{66,67}$. In addition, the epithelial-to-mesenchymal transition (EMT) is one of the mechanisms leading to apoptotic failure and drug resistance in epithelial-derived carcinoma cells ${ }^{68}$. Evidence has indicated that tumour cells in a high-mesenchymal state are characterised by enhanced activity of enzymes related to the promotion of PUFA synthesis, making these cells dependent on the lipid peroxidase pathway involving GPX4. Thus, cancer cells in a mesenchymal state can undergo ferroptosis through pharmacological perturbations to overcome cancer therapy resistance ${ }^{68}$. Targeting ferroptosis is a new perspective for the treatment of kidney injury, non-cancer liver diseases, and intestinal diseases, suggesting the significant potential of ferroptosis research ${ }^{10,69}$.

\section{Ferroptosis in intestinal disease}

Ferroptosis and intestinal ischaemia/reperfusion (I/R) injury

Intestinal I/R injury is a common clinical condition with high morbidity and mortality, resulting from sudden reduction of intestinal blood flow and reoxygenation after the restoration of blood supply ${ }^{70}$. It occurs in many clinical conditions, including trauma, haemorrhagic shock, acute mesenteric ischaemia, small intestinal volvulus, and intestinal transplantation ${ }^{71,72}$. Intestinal mucosal barrier dysfunction, as a consequence of epithelial cell death, can allow the translocation of bacteria and associated toxins into the bloodstream, leading to inflammation, systemic sepsis, and organ dysfunction ${ }^{73,74}$. Intestinal I/R injury is associated with multiple types of RCD, including apoptosis, necroptosis, and autophagy, but with the discovery of ferroptosis, new potential mechanisms of RCD have attracted attention $^{75-77}$. Indeed, ferroptosis has been identified in $\mathrm{I} / \mathrm{R}$ injury in other organs both in vivo and in vitro; moreover, ferroptosis inhibitors can alleviate these injuries. Studies have shown that treatment with DFO reduced myocardial infarct size and lactate dehydrogenase levels in an ex vivo heart model of I/R stress ${ }^{9}$, while Lip-1 prevented acute renal failure from renal $I / R$ injury ${ }^{39}$. ROS generation and lipid peroxidation are associated with intestinal $I / R$ injury and are primary contributors to the initiation and execution of ferroptosis ${ }^{78}$. Decreased GSH levels and superoxide dismutase activity, as well as increased MDA levels, were observed in rat intestinal tissues after intestinal $I / R^{79,80}$. Furthermore, DFO administration was beneficial in the prevention of intestinal I/R-induced lipid peroxidation and GPX activity reduction was reversed by DFO treatment ${ }^{81}$. Taken together, lipid peroxidation and iron participate in $\mathrm{I} /$ R-induced intestinal injury, but their contribution to ferroptosis is still enigmatic.
A recent study has reported that ferroptosis plays a critical role in intestinal I/R injury and may be a lethal process triggered by reperfusion ${ }^{14}$. In this study, the expression levels of pro-ferroptotic factors such as ACSL4 and iron increased, while those of anti-ferroptotic factors (GPX4, FTH1, GSH) decreased in ischaemic murine intestinal tissues; moreover, treatment with Lip-1 ameliorated intestinal injury both in vivo and in vitro ${ }^{14}$. Moreover, an ischaemia model that incorporated different reperfusion durations to examine features of ferroptosis in situ suggested that this form of cell death occurred in the early phase of reperfusion and was distinct from apoptosis, which appeared at a later phase ${ }^{14}$. Inhibition of ischaemia-induced ACSL4 (a key regulator and indicator of ferroptosis) expression via pharmacological and genetic manipulations protected against lipid peroxidation and ferroptosis, as well as alleviated cell damage and intestinal barrier dysfunction induced by intestinal $I / R^{14,25} . \mathrm{Li}$ and colleagues have further shown that Sp1, a transcription factor that binds to GC-box motifs in target-gene promoters, mediated ACSL4 expression ${ }^{14}$. In addition to intestinal damage, intestinal $\mathrm{I} / \mathrm{R}$ can cause acute injury to remote organs, including the lungs and liver. Indeed, ferroptosis was reported to exacerbate intestinal I/Rinduced acute lung injury, whereas blocking this process mitigated lung injury after intestinal $I / R$ in mouse models $^{14,82}$. In summary, ferroptosis is related to I/R-induced intestinal injury, but more studies are needed to discover its underlying mechanisms and regulation.

\section{Ferroptosis and inflammatory bowel disease}

Inflammatory bowel disease (IBD), including ulcerative colitis (UC) and Crohn's disease (CD), is a chronic disease characterised by constant progression and relapse. Although not fully elucidated, the aetiology of IBD is commonly thought to implicate reciprocal interactions between host genetics, environmental factors, the gut microbiome, and immune responses ${ }^{83}$. A better understanding of IBD pathogenesis will be beneficial for improving its treatment; recent studies have underlined the importance of cell death in intestinal epithelial homoeostasis. Excessive cell death is closely correlated with chronic inflammation in IBD patients ${ }^{84}$, but what is the relationship between ferroptosis and IBD? It has been reported that iron supplementation changes gut microbial homoeostasis and exacerbates intestinal inflammation similarly to $\mathrm{CD}$ in a murine model ${ }^{85}$. Another study using a rat model of DSS-induced colitis also indicated that excess iron aggravated intestinal inflammation ${ }^{86}$. Recently, a Japanese team showed that high dietary iron intake increased the risk of $\mathrm{UC}^{87}$. Mucosal ROS production is increased in UC in proportion to the disease activity, and iron chelators are known to reduce ROS production and ameliorate colonic symptoms in $\mathrm{IBD}^{88,89}$. 
Taken together, these findings suggest a possible relationship between IBD and ferroptosis in which excess iron in the intestine produces ROS via the Fenton reaction, which triggers oxidative stress. Lipid peroxidation procedurally appears and ferroptotic cell death is induced. Thereby, the intestinal epithelial cells are destroyed, and damage to the intestinal mucosal barrier results in $\mathrm{IBD}^{90}$.

Ferroptosis has been implicated in both clinical UC patients and in murine experimental colitis, with significant downregulation and upregulation of ferroptosis-associated genes $^{12,13}$. Administration of ferroptosis inhibitors, including Fer-1, Lip-1, and DFO, reduced disease activity scores and ameliorated colon length shortening in DSSinduced murine colitis, suggesting the beneficial effect of inhibiting ferroptosis ${ }^{12,13,91}$. In keeping with ferroptosis mechanisms, GPX4 also plays a vital role in negatively regulating ferroptotic cell death in IBD. Curculigoside (CUR) is a botanical ingredient with anti-oxidant and antiinflammatory properties that protects against ferroptosis in UC through the promotion of GPX $4^{91}$. CUR increased selenium sensitivity and enhance GPX4 expression levels in the IEC-6 rat intestinal epithelial cell line, while Gpx4 silencing inhibited the protective effects of CUR on cell death and oxidative stress indicators in ferroptotic IEC-6 cells $^{91}$. Moreover, another group emphasised the importance of GPX4 in gut homoeostasis by showing impaired GPX4 activity and features of lipid peroxidation in the small intestinal epithelial cells (IECs) of CD patients ${ }^{92}$. PUFAs, especially AA, induced the production of interleukin 6 (IL-6) and chemokine (C-X-C motif) ligand 1 (CXCL1) in IECs treated with Gpx4 siRNA in response to iron availability, lipid peroxidation, and ACSL4, similar to ferroptosis mechanisms ${ }^{92}$. Interestingly, a PUFA-enriched Western diet triggered small intestinal inflammation in mice that lacked one Gpx4 allele in IECs, with histological characteristics resembling $\mathrm{CD}^{92}$. The link between PUFA uptake, GPX4 activity, and intestinal inflammation further provides evidence for the pathogenesis of $\mathrm{CD}$. However, although the process observed in the study was similar to ferroptosis, no definite cell death was observed in the murine intestinal inflammation model; the scholars speculate that in this case, one Gpx4 allele might be sufficient to protect against ferroptotic cell death ${ }^{92}$.

NRF2 is a critical mediator of the cellular antioxidant response that controls redox homoeostasis-related gene expression; perturbations of the NRF2-lipid peroxidation-ferroptosis axis have been found in cancers $^{63}$. HO-1, a cytoprotective enzyme related to cellular stress, also participates in ferroptosis and has antiinflammatory effects ${ }^{93}$. Chen et al. found that Fer-1 alleviated DSS-induced colitis via NRF2/HO-1 signalling, indicating that the NRF2 pathway may be an important factor regulating ferroptosis in $\mathrm{UC}^{12}$. ER stress can induce the cell-death signalling pathway in the form of apoptosis and autophagy ${ }^{94,95}$, but interestingly, ER stress also is implicated in the development of ferroptosis in diseases, including $\mathrm{IBD}^{13,96}$. It has been found that ferroptosis contributed to UC via ER stress-mediated IEC cell death; moreover, phosphorylation of NF-kBp65 inhibited ER stress-mediated IEC ferroptosis to relieve the disease as an upstream regulator ${ }^{13}$. Together, these data show that ferroptosis has a key role in IBD, and that targeting it may be a promising method for understanding the development of IBD and to provide new treatments.

\section{Ferroptosis and colorectal cancer}

Colorectal cancer (CRC) is a common malignant tumour with high morbidity and mortality and is one of the most pressing global health issues. According to the GLOBOCAN 2018 estimates of incidence and mortality worldwide report, CRC is the third-most diagnosed cancer and the second-most cause of cancer-related deaths globally ${ }^{97}$. Current treatments for CRC include surgery, radiotherapy, chemotherapy, immune therapy, and biotargeted therapy ${ }^{98}$; however, despite recent progress in therapeutics, some patients exhibit resistance or intolerance to them via apoptosis evasion and anti-apoptotic enhancement ${ }^{99,100}$. Thus ferroptosis, as a form of RCD independent from apoptosis, may provide a promising strategy for cancer therapy. Since its discovery in 2012, the manipulation of ferroptosis by specific molecules has enabled inhibition of the growth and spread of multiple cancer types, including $\mathrm{CRC}^{1,23}$. RSL3-induced ferroptosis in a dose-and time-dependent manner in three CRC cell lines; this treatment increased ROS and cellular labile iron pool (LIP) levels ${ }^{17}$. Evidence has showed that the classic chemotherapy drug cisplatin induces ferroptosis; moreover, the combination of cisplatin and erastin was synergistic, indicating that ferroptosis adds an alternative cell-death pathway triggered by classical therapeutic drugs and antitumour mechanisms in $\mathrm{CRC}^{100}$. In addition, targeting ferroptosis can overcome conventional CRC drug resistance from a new perspective. Chen et al. reported that the bioactive compound $\beta$-elemene (extracted from the Chinese herb Curcumae Rhizoma) is a ferroptosis inducer; they combined treatment with $\beta$-elemene and anti-EGFR (epidermal growth factor receptor) antibody cetuximab to produce anti-tumour effects by triggering ferroptosis in CRC patients with RAS mutations that do not respond to cetuximab $^{99,101}$. Another study showed that vitamin $C$, an antioxidant that can paradoxically initiate oxidative stress at pharmacological doses, targeted cetuximab-persister cells and restricted the emergence of acquired resistance to EGFR blockade in CRC through the induction of ferropto$\mathrm{sis}^{67}$. Altogether, the role of ferroptosis in CRC in inhibiting tumour growth and overcoming resistance to current anticancer drugs is a promising avenue for research. 
As described above, GPX4 plays a central role in regulating ferroptosis. Recently, several molecules have been implicated in ferroptosis in CRC through their mediation of GPX4. RSL3 inhibits GPX4 activity by directly binding with GPX4, and overexpression of GPX4 rescued CRC cell death induced by RSL3, suggesting a similar role of GPX4 in RSL3-induced ferroptosis in CRC as in other diseases ${ }^{17}$. Shen et al. found that resibufogenin isolated from Asiatic toad dried skin secretions is a potential anticancer agent in the treatment of CRC because it induced ferroptosis in a GPX4 inactivation-dependent manner ${ }^{18}$. In addition to direct GPX4 inhibition, inhibiting SLC7A11, the functional subunit of system $\mathrm{X}_{\mathrm{c}}{ }^{-}$, also induces ferroptotic cell death in CRC. It was reported that the benzopyran derivative 2-imino-6-methoxy-2H-chromene-3-carbothioamide (IMCA) has a wide spectrum of biological activities, including those relevant to cancer therapy ${ }^{102}$. Zhang et al. first discovered the anti-CRC effect of IMCA through ferroptosis induction by downregulating SLC7A11 ${ }^{103}$. Interestingly, IMCA affected the downstream components of the AMPK/mTOR/p70S6k pathway, which have been linked to SLC7A11 activity and ferroptosis ${ }^{103}$. The role of SLC7A11 and ferroptosis has also been elucidated in colorectal cancer stem cells (CSCs), which can provide resistance to chemotherapy and form secondary tumours in the progression of CRC ${ }^{104}$. Colorectal CSCs are more sensitive to ferroptosis than parental CRC cells; the knockout of SLC7A11 with CRISPR-Cas9 technology facilitated ferroptotic cell death, suggesting that targeting SLC7A11 may specifically suppress the progression of colorectal CSCs and reduce CRC drug resistance ${ }^{105}$. Another key regulator of ferroptosis in many related diseases is $\mathrm{ACSL}^{25}$. A recent study has determined its crucial regulatory role in the induction of ferroptosis by bromelain (a plant extract derived from pineapple) in KRAS-mutant CRC through signalling pathway and miRNA profiling ${ }^{106}$.

The TP53 gene is known as a tumour suppressor and ferroptosis regulator in multiple cancers. It inhibited ferroptotic CRC cell death by blocking dipeptidyl-peptidase4 (DPP4) activity, while the loss of p53 increased the anticancer activity of erastin in tumour-bearing mice, very different from the positive regulation of ferroptosis by $\mathrm{p} 53$ in other cancers (Fig. 3) ${ }^{61}$. Specifically, the loss of p53 restrains DPP4 nuclear localisation and facilitates the formation of the DPP4-NOX1 complex that promotes lipid peroxidation, resulting in ferroptosis in the HCT116 human CRC cell line ${ }^{59,61}$. While p53 can limit ferroptosis by forming a DPP4-p53 complex in the nucleus, disassembly of this complex restores the erastin-induced ferroptosis sensitivity of $C R C^{59,61}$. Moreover, the fact that TP53 can stimulate SLC7A11 expression in CRC protects CRC cells from ferroptosis ${ }^{61}$. Therefore, regulation of TP53 may be highly desirable as part of CRC therapy. In a human miRNome analysis of miRNA-mRNA interactions and multiple pathways involved in CRC pathogenesis, three downregulated miRNAs, let-7c, let-7e, and miR150-5p, were found to modulate TP53 in CRC and thus could regulate ferroptosis ${ }^{107}$.

There also are connections between ferroptosis and other types of RCDs in CRC. Hong et al. reported molecular crosstalk between ferroptosis and apoptosis when CRC cells were treated with the ferroptotic agents erastin or artesunate (ART) in combination with the apoptotic agent tumour necrosis factor-related apoptosis-inducing ligand (TRAIL) ${ }^{108}$. The combination of erastin/ART and TRAIL significantly promoted TRAIL-induced apoptosis due to ER stress-induced p53-independent PUMA (p53 upregulated modulator of apoptosis) expression ${ }^{108}$. The group further found that ER stress response-mediated expression of the TRAIL receptor death receptor 5 (DR5) also played a vital role in this combinatorial synergy in a variety of CRC cell lines ${ }^{109}$. These studies are preliminary explorations of the mechanisms that may be shared between ferroptosis and apoptosis, but further research is needed to understand the relationship between these RCDs. Autophagy promotes ferroptotic cell death through the degradation of ferritin (ferritinophagy) in fibroblasts and certain cancer cells, modulated by selective cargo receptor $\mathrm{NCOA} 4^{54,55}$; its inhibition suppresses ferritin degradation and inhibits ferroptosis in these cells ${ }^{54,55}$. However, this is not the case for CRC cells; Hasan et al. indicated that ferritinophagy was not required for CRC cell growth ${ }^{110}$. Interestingly, knocking out NCOA4 did not alter ferroptosis in CRC ${ }^{110}$. Differences in cell lines may partially explain these conflicting findings, but another possibility is that CRC cells have an alternative mechanism that compensates for the loss of NCOA4 function in response to ferroptosis induction. Future studies should investigate the compensatory and alternative pathways in CRC that enhance cell survival.

Taken together, ferroptosis plays a significant role in $\mathrm{CRC}$, and its regulation may provide new insights into cancer therapy. In addition to the regulators already mentioned, researchers also have identified novel compounds, for example, iron oxide-hydroxide nanospindles, with the potential to promote ferroptosis to inhibit colon cancer ${ }^{111}$. Researchers also have identified new targets which can regulate ferroptosis in CRC, including the short/branched chain acyl-coenzyme A dehydrogenase and anoctamin $6^{112,113}$. We believe that fully understanding ferroptosis and its underlying mechanisms in $\mathrm{CRC}$, as well as the connections between ferroptosis and other RCDs, can give us hope to improve the treatment and prognosis of this cancer.

\section{Conclusions and perspectives}

Ferroptosis is a newly identified type of RCD that is mediated by the iron-dependent accumulation of lipid 
ROS and has been implicated in the development of a wide variety of disorders, especially intestinal diseases. Inhibiting ferroptosis can attenuate intestinal injury in non-infectious inflammatory conditions such as intestinal $I / R$ injury and IBD, while inducing ferroptosis with pharmacological activators can inhibit the migration, invasion, and proliferation of colorectal neoplasms, suggesting a dual role for ferroptosis in different intestinal diseases. As shown in Fig. 2, the common ferroptotic mechanisms in intestinal diseases include GPX4 inhibition, system $\mathrm{X}_{\mathrm{c}}{ }^{-}$suppression, lipid peroxide accretion, and iron overload, which are consistent with other diseases. Key regulators such as GPX4, SLC7A11, ACSL4, and p53 are also important for mediating ferroptosisassociated intestinal diseases. In addition to known ferroptotic inducers (erastin, RSL3) and inhibitors (Fer-1, Lip-1, DFO), researchers have found more drugs and targets related to ferroptosis in intestinal diseases (Table 1). However, whether there are specific regulators or signalling pathways in these diseases remains unclear. Interestingly, some regulators seem to play different roles in intestinal diseases compared with diseases in other organs, for example, p53 and NCOA4 in CRC. As a result, further research is needed to identify disease-specific ferroptotic mechanisms to develop disease contextdependent therapeutic regimens. Furthermore, studies have found correlations between ferroptosis and other forms of cell death in intestinal diseases. These RCDs may share common pathways and key regulators, which can provide new directions for combining different therapeutic interventions.

Although much progress has been made, research on intestinal ferroptosis is still at an early stage, and its specific role remains to be investigated across the spectrum of intestinal disease, including many not presented in this review. Although we have summarized multiple methods for assaying ferroptosis from multiple aspects, no unanimously agreed-upon criteria directly define its occurrence. It is imperative to identify markers and other approaches to assess ferroptosis in vivo. In this way, ferroptosis biomarkers could be promising to indicate intestinal disease severity. We caution that the relationship between ferroptotic cell death and iron/lipid peroxides remains controversial, so more evidence is required to support the links between ferroptosis and iron, oxidative stress, and lipid peroxidation in disease development and progression. In addition, the signalling pathways and main transcriptional regulators of ferroptosis need to be studied so that we can benefit more from its modulation to protect the intestine against injury and carcinogenesis. Therefore, ferroptosis should be further investigated within the field of intestinal disease as a novel therapeutic target.

\section{Acknowledgements}

We would like to thank Editage (www.editage.cn) for English language editing.

\section{Author contributions}

Guarantor of the article: S.Z. S.Z. and M.C. designed and oversaw the study. S.X. and Y.H. wrote and revised the manuscript. S.Z., M.C., P.C., and L.L. revised the contents of the manuscript. All authors approved the final manuscript.

\section{Funding}

This work was supported by grants from the National Natural Science Foundation of China (\#81630018, \#82070538, \#81870374), Guangzhou Science and Technology Department (\#202002030041), Guangdong Science and Technology Department (\#2017A030306021), and the Fundamental Research Funds for the Central Universities (\#19ykzd11).

\section{Ethics}

This paper has been approved by the Medical Ethics Committee of the First Affiliated Hospital, Sun Yat-sen University.

\section{Conflict of interest}

The authors declare no competing interests.

\section{Publisher's note}

Springer Nature remains neutral with regard to jurisdictional claims in published maps and institutional affiliations.

Received: 12 October 2020 Revised: 23 February 2021 Accepted: 24 February 2021

Published online: 17 March 2021

\section{References}

1. Dixon, S. J. et al. Ferroptosis: an iron-dependent form of nonapoptotic cell death. Cell 149, 1060-1072 (2012).

2. Stockwell, B. R. et al. Ferroptosis: a regulated cell death nexus linking metabolism, redox biology, and disease. Cell 171, 273-285 (2017).

3. Dixon, S. J. \& Stockwell, B. R. The role of iron and reactive oxygen species in cell death. Nat. Chem. Biol. 10, 9-17 (2014).

4. Xie, Y. et al. Ferroptosis: process and function. Cell Death Differ. 23, 369-379 (2016).

5. Yang, W. S. et al. Regulation of ferroptotic cancer cell death by GPX4. Cell 156, 317-331 (2014).

6. Cao, J. Y. \& Dixon, S. J. Mechanisms of ferroptosis. Cell Mol. Life Sci. 73, 2195-2209 (2016)

7. Imai, H., Matsuoka, M., Kumagai, T., Sakamoto, T. \& Koumura, T. Lipid peroxidation-dependent cell death regulated by GPx4 and ferroptosis. Curr. Top. Microbiol. Immunol. 403, 143-170 (2017).

8. Angeli, J. P. F., Shah, R., Pratt, D. A. \& Conrad, M. Ferroptosis inhibition: mechanisms and opportunities. Trends Pharm. Sci. 38, 489-498 (2017).

9. Gao, M., Monian, P., Quadri, N., Ramasamy, R. \& Jiang, X. Glutaminolysis and transferrin regulate ferroptosis. Mol. Cell 59, 298-308 (2015).

10. Hu, Z. et al. Emerging role of ferroptosis in acute kidney injury. Oxid. Med Cell Longev. 2019, 8010614 (2019).

11. Del, Re. D. P., Amgalan, D., Linkermann, A., Liu, Q. \& Kitsis, R. N. Fundamental mechanisms of regulated cell death and implications for heart disease. Physiol. Rev. 99, 1765-1817 (2019).

12. Chen, Y., Zhang, P., Chen, W. \& Chen, G. Ferroptosis mediated DSS-induced ulcerative colitis associated with $\mathrm{Nrf} 2 / \mathrm{HO}-1$ signaling pathway. Immunol. Lett. 225, 9-15 (2020).

13. Xu, M. et al. Ferroptosis involves in intestinal epithelial cell death in ulcerative colitis. Cell Death Dis. 11, 86 (2020).

14. Li, Y. et al. Ischemia-induced ACSL4 activation contributes to ferroptosismediated tissue injury in intestinal ischemia/reperfusion. Cell Death Differ. 26, 2284-2299 (2019).

15. Song, Y., Yang, H., Lin, R., Jiang, K. \& Wang, B. M. The role of ferroptosis in digestive system cancer. Oncol. Lett. 18, 2159-2164 (2019).

16. Nie, J., Lin, B., Zhou, M., Wu, L. \& Zheng, T. Role of ferroptosis in hepatocellular carcinoma. J. Cancer Res. Clin. Oncol. 144, 2329-2337 (2018). 
17. Sui, $X$. et al. RSL3 drives ferroptosis through GPX4 inactivation and ROS production in colorectal cancer. Front Pharm. 9, 1371 (2018).

18. Shen, L. D. et al. Resibufogenin inhibited colorectal cancer cell growth and tumorigenesis through triggering ferroptosis and ROS production mediated by GPX4 inactivation. Anat. Rec. (Hoboken) 304, 313-322 (2021).

19. Dolma, S., Lessnick, S. L., Hahn, W. C. \& Stockwell, B. R. Identification of genotype-selective antitumor agents using synthetic lethal chemical screening in engineered human tumor cells. Cancer Cell 3, 285-296 (2003)

20. Yang, W. S. \& Stockwell, B. R. Synthetic lethal screening identifies compounds activating iron-dependent, nonapoptotic cell death in oncogenic-RASharboring cancer cells. Chem. Biol. 15, 234-245 (2008).

21. Capelletti, M. M., Manceau, H., Puy, H. \& Peoc'h, K. Ferroptosis in liver diseases: an overview. Int. J. Mol. Sci. 21, 4908 (2020).

22. Galluzzi, L. et al. Molecular mechanisms of cell death: recommendations of the Nomenclature Committee on Cell Death 2018. Cell Death Differ. 25 486-541 (2018).

23. Li, J. et al. Ferroptosis: past, present and future. Cell Death Dis. 11, 88 (2020).

24. Jiang, $L$. et al. Ferroptosis as a p53-mediated activity during tumour suppression. Nature 520, 57-62 (2015).

25. Doll, $\mathrm{S}$. et al. ACSL4 dictates ferroptosis sensitivity by shaping cellular lipid composition. Nat. Chem. Biol. 13, 91-98 (2017).

26. Sun, X. et al. Activation of the p62-Keap1-NRF2 pathway protects against ferroptosis in hepatocellular carcinoma cells. Hepatology 63, 173-184 (2016).

27. Yu, Y. et al. The ferroptosis inducer erastin enhances sensitivity of acute myeloid leukemia cells to chemotherapeutic agents. Mol. Cell Oncol. 2, e1054549 (2015)

28. Tyurin, V. A. et al. Oxidative lipidomics of programmed cell death. Methods Enzymol. 442, 375-393 (2008)

29. Wiernicki, B. et al. Excessive phospholipid peroxidation distinguishes ferroptosis from other cell death modes including pyroptosis. Cell Death Dis. 11, 922 (2020)

30. Drummen, G. P., van Liebergen, L. C., Op den Kamp, J. A. \& Post, J. A. C11BODIPY(581/591), an oxidation-sensitive fluorescent lipid peroxidation probe: (micro)spectroscopic characterization and validation of methodology. Free Radic. Biol. Med. 33, 473-490 (2002).

31. Ayala, A., Muñoz, M. F. \& Argüelles, S. Lipid peroxidation: production, metabolism, and signaling mechanisms of malondialdehyde and 4-hydroxy-2nonenal. Oxid. Med. Cell Longev. 2014, 360438 (2014).

32. Xian, Z. Y. et al. CircABCB10 silencing inhibits the cell ferroptosis and apoptosis by regulating the miR-326/CCL5 axis in rectal cancer. Neoplasma 67, 1063-1073 (2020)

33. Mou, $Y$. et al. Ferroptosis, a new form of cell death: opportunities and challenges in cancer. J. Hematol. Oncol. 12, 34 (2019).

34. Doll, S. \& Conrad, M. Iron and ferroptosis: a still ill-defined liaison. IUBMB Life 69, 423-434 (2017).

35. Kajarabille, N. \& Latunde-Dada, G. O. Programmed cell-death by ferroptosis: antioxidants as mitigators. Int. J. Mol. Sci. 20, 4968 (2019).

36. Martin, H. L. \& Teismann, P. Glutathione-a review on its role and significance in Parkinson's disease. FASEB J. 23, 3263-3272 (2009).

37. Ursini, F., Maiorino, M., Valente, M., Ferri, L. \& Gregolin, C. Purification from pig liver of a protein which protects liposomes and biomembranes from peroxidative degradation and exhibits glutathione peroxidase activity on phosphatidylcholine hydroperoxides. Biochim. Biophys. Acta 710, 197-211 (1982).

38. Brigelius-Flohé, R. \& Maiorino, M. Glutathione peroxidases. Biochim. Biophys. Acta 1830, 3289-3303 (2013).

39. Friedmann Angeli, J. P. et al. Inactivation of the ferroptosis regulator Gpx4 triggers acute renal failure in mice. Nat. Cell Biol. 16, 1180-1191 (2014).

40. Liang, C., Zhang, X., Yang, M. \& Dong, X. Recent progress in ferroptosis inducers for cancer therapy. Adv. Mater. 31, e1904197 (2019).

41. Yang, W. S. et al. Peroxidation of polyunsaturated fatty acids by lipoxygenases drives ferroptosis. Proc. Natl Acad. Sci. USA 113, E4966-E4975 (2016).

42. Ursini, F. \& Maiorino, M. Lipid peroxidation and ferroptosis: The role of GSH and GPx4. Free Radic. Biol. Med. 152, 175-185 (2020).

43. Lu, S. C. Glutathione synthesis. Biochim Biophys. Acta 1830, 3143-3153 (2013).

44. Bridges, R. J., Natale, N. R. \& Patel, S. A. System $X_{c}{ }^{-}$cystine/glutamate antiporter: an update on molecular pharmacology and roles within the CNS. Br. J. Pharm. 165, 20-34 (2012).

45. McBean, G. J. The transsulfuration pathway: a source of cysteine for glutathione in astrocytes. Amino Acids 42, 199-205 (2012).
46. Yuan, H., Li, X., Zhang, X., Kang, R. \& Tang, D. Identification of ACSL4 as a biomarker and contributor of ferroptosis. Biochem. Biophys. Res. Commun. 478, 1338-1343 (2016)

47. Dixon, S. J. et al. Human haploid cell genetics reveals roles for lipid metabolism genes in nonapoptotic cell death. ACS Chem. Biol. 10, 1604-1609 (2015).

48. Kagan, V. E. et al. Oxidized arachidonic and adrenic PEs navigate cells to ferroptosis. Nat. Chem. Biol. 13, 81-90 (2017).

49. Feng, H. \& Stockwell, B. R. Unsolved mysteries: How does lipid peroxidation cause ferroptosis? PLoS Biol. 16, e2006203 (2018).

50. Stoyanovsky, D. A. et al. Iron catalysis of lipid peroxidation in ferroptosis: regulated enzymatic or random free radical reaction? Free Radic. Biol. Med. 133, 153-161 (2019).

51. Lane, D. J. et al. Cellular iron uptake, trafficking and metabolism: key molecules and mechanisms and their roles in disease. Biochim. Biophys. Acta 1853 1130-1144 (2015).

52. Coffey, R. \& Ganz, T. Iron homeostasis: an anthropocentric perspective. J. Biol. Chem. 292, 12727-12734 (2017).

53. Kwon, M. Y., Park, E., Lee, S. J. \& Chung, S. W. Heme oxygenase-1 accelerates erastin-induced ferroptotic cell death. Oncotarget 6, 24393-24403 (2015).

54. Gao, M. et al. Ferroptosis is an autophagic cell death process. Cell Res. 26 1021-1032 (2016).

55. Hou, W. et al. Autophagy promotes ferroptosis by degradation of ferritin. Autophagy 12, 1425-1428 (2016)

56. Brown, C. W. et al. Prominin2 drives ferroptosis resistance by stimulating iron export. Dev. Cell 51, 575-586 (2019).

57. Sun, $X$. et al. HSPB1 as a novel regulator of ferroptotic cancer cell death. Oncogene 34, 5617-5625 (2015).

58. Yuan, H., Li, X., Zhang, X., Kang, R. \& Tang, D. CISD1 inhibits ferroptosis by protection against mitochondrial lipid peroxidation. Biochem. Biophys. Res. Commun. 478, 838-844 (2016).

59. Zhang, W., Gai, C., Ding, D., Wang, F. \& Li, W. Targeted p53 on smallmolecules-induced ferroptosis in cancers. Front Oncol. 8, 507 (2018).

60. Tarangelo, A. et al. p53 suppresses metabolic stress-induced ferroptosis in cancer cells. Cell Rep. 22, 569-575 (2018).

61. Xie, Y. et al. The tumor suppressor p53 limits ferroptosis by blocking DPP4 activity. Cell Rep. 20, 1692-1704 (2017).

62. Doll, S. et al. FSP1 is a glutathione-independent ferroptosis suppressor. Nature 575, 693-698 (2019).

63. Dodson, M., Castro-Portuguez, R. \& Zhang, D. D. NRF2 plays a critical role in mitigating lipid peroxidation and ferroptosis. Redox Biol. 23, 101107 (2019).

64. Xu, T. et al. Molecular mechanisms of ferroptosis and its role in cancer therapy. J. Cell Mol. Med. 23, 4900-4912 (2019).

65. Oxnard, G. R. The cellular origins of drug resistance in cancer. Nat. Med. 22, 232-234 (2016).

66. Hangauer, M. J. et al. Drug-tolerant persister cancer cells are vulnerable to GPX4 inhibition. Nature 551, 247-250 (2017)

67. Lorenzato, $\mathrm{A}$. et al. Vitamin $\mathrm{C}$ restricts the emergence of acquired resistance to EGFR-targeted therapies in colorectal cancer. Cancers (Basel) 12, 685 (2020).

68. Viswanathan, V. S. et al. Dependency of a therapy-resistant state of cancer cells on a lipid peroxidase pathway. Nature 547, 453-457 (2017).

69. Mao, L. et al. The emerging role of ferroptosis in non-cancer liver diseases: hype or increasing hope? Cell Death Dis. 11, 518 (2020).

70. Du, L., Zhang, R., Luo, T., Nie, M. \& Bi, J. Effects of helium preconditioning on intestinal ischemia and reperfusion injury in rats. Shock 44, 365-370 (2015).

71. Mallick, I. H., Yang, W., Winslet, M. C. \& Seifalian, A. M. Ischemia-reperfusion injury of the intestine and protective strategies against injury. Dig. Dis. Sci. 49, 1359-1377 (2004).

72. Gonzalez, L. M., Moeser, A. J. \& Blikslager, A. T. Animal models of ischemiareperfusion-induced intestinal injury: progress and promise for translationa research. Am. J. Physiol. Gastrointest. Liver Physiol. 308, G63-G75 (2015).

73. Cheng, J. et al. The role of intestinal mucosa injury induced by intraabdominal hypertension in the development of abdominal compartment syndrome and multiple organ dysfunction syndrome. Crit. Care 17, R283 (2013).

74. Berg, R. D. Bacterial translocation from the gastrointestinal tract. Trends Microbiol. 3, 149-154 (1995).

75. Wang, G. et al. miR-34a-5p inhibition alleviates intestinal ischemia/reperfusion-induced reactive oxygen species accumulation and apoptosis via activation of SIRT1 signaling. Antioxid. Redox Signal 24, 961-973 (2016). 
76. Wen, S. et al. Necroptosis is a key mediator of enterocytes loss in intestinal ischaemia/reperfusion injury. J. Cell Mol. Med. 21, 432-443 (2017).

77. Li, Z. et al. Targeting the miR-665-3p-ATG4B-autophagy axis relieves inflammation and apoptosis in intestinal ischemia/reperfusion. Cell Death Dis. 9, 483 (2018)

78. Hu, Y. et al. Protective effect of dioscin against intestinal ischemia/reperfusion injury via adjusting miR-351-5p-mediated oxidative stress. Pharm. Res. 137, 56-63 (2018).

79. Stefanutti, G., Pierro, A., Parkinson, E. J., Smith, V. V. \& Eaton, S. Moderate hypothermia as a rescue therapy against intestinal ischemia and reperfusion injury in the rat. Crit. Care Med. 36, 1564-1572 (2008).

80. Ozkan, O. V. et al. Resveratrol, a natural antioxidant, attenuates intestinal ischemia/reperfusion injury in rats. Tohoku J. Exp. Med. 218, 251-258 (2009).

81. Balogh, N. et al. Effect of deferoxamine and L-arginine treatment on lipid peroxidation in an intestinal ischaemia-reperfusion model in rats. Acta. Vet. Hung. 50, 343-356 (2002)

82. Li, Y. et al. Inhibitor of apoptosis-stimulating protein of p53 inhibits ferroptosis and alleviates intestinal ischemia/reperfusion-induced acute lung injury. Cell Death Differ. 27, 2635-2650 (2020).

83. Sartor, R. B. \& Wu, G. D. Roles for intestinal bacteria, viruses, and fungi in pathogenesis of inflammatory bowel diseases and therapeutic approaches. Gastroenterology 152, 327-339 (2017).

84. Günther, C., Neumann, H., Neurath, M. F. \& Becker, C. Apoptosis, necrosis and necroptosis: cell death regulation in the intestinal epithelium. Gut $\mathbf{6 2}$, 1062-1071 (2013).

85. Werner, T. et al. Depletion of luminal iron alters the gut microbiota and prevents Crohn's disease-like ileitis. Gut 60, 325-333 (2011).

86. Carrier, J. C., Aghdassi, E., Jeejeebhoy, K. \& Allard, J. P. Exacerbation of dextran sulfate sodium-induced colitis by dietary iron supplementation: role of NFkappaB. Int. J. Colorectal Dis. 21, 381-387 (2006).

87. Kobayashi, Y. et al. Association between dietary iron and zinc intake and development of ulcerative colitis: a case-control study in Japan. J. Gastroenterol. Hepatol. 34, 1703-1710 (2019).

88. Millar, A. D., Rampton, D. S. \& Blake, D. R. Effects of iron and iron chelation in vitro on mucosal oxidant activity in ulcerative colitis. Aliment Pharm. Ther. 14, 1163-1168 (2000).

89. Minaiyan, M., Mostaghel, E. \& Mahzouni, P. Preventive therapy of experimental colitis with selected iron chelators and anti-oxidants. Int. J. Prev. Med. 3, S162-S169 (2012)

90. Qi, X. et al. Mechanism and intervention measures of iron side effects on the intestine. Crit. Rev. Food Sci. Nutr. 60, 2113-2125 (2020).

91. Wang S., Liu W., Wang J. \& Bai X. Curculigoside inhibits ferroptosis in ulcerative colitis through the induction of GPX4. Life Sci. 259, 118356 (2020),

92. Mayr, L. et al. Dietary lipids fuel GPX4-restricted enteritis resembling Crohn's disease. Nat. Commun. 11, 1775 (2020).

93. Adedoyin, O. et al. Heme oxygenase-1 mitigates ferroptosis in renal proximal tubule cells. Am. J. Physiol. Ren. Physiol. 314, F702-F714 (2018).

94. Zeng, L. X. et al. $\beta$-Arrestin2 encourages inflammation-induced epithelial apoptosis through ER stress/PUMA in colitis. Mucosal Immunol. 8, 683-695 (2015).
95. Bhardwaj, M., Leli, N. M., Koumenis, C. \& Amaravadi, R. K. Regulation of autophagy by canonical and non-canonical ER stress responses. Semin Cancer Biol. 66, 116-128 (2020).

96. Park, E. J., Park, Y. J., Lee, S. J., Lee, K. \& Yoon, C. Whole cigarette smoke condensates induce ferroptosis in human bronchial epithelial cells. Toxicol. Lett. 303, 55-66 (2019).

97. Bray, F. et al. Global cancer statistics 2018: GLOBOCAN estimates of incidence and mortality worldwide for 36 cancers in 185 countries. CA Cancer J. Clin. $\mathbf{6 8}$, 394-424 (2018).

98. Benson, A. B. et al. NCCN Guidelines Insights: Colon Cancer, Version 2.2018. J. Natl. Compr. Canc. Netw. 16, 359-369 (2018).

99. Chen, $P$. et al. Combinative treatment of $\beta$-elemene and cetuximab is sensitive to KRAS mutant colorectal cancer cells by inducing ferroptosis and inhibiting epithelial-mesenchymal transformation. Theranostics 10, 5107-5119 (2020).

100. Guo, J. et al. Ferroptosis: A novel anti-tumor action for cisplatin. Cancer Res. Treat. 50, 445-460 (2018).

101. Serebriiskii, I. G. et al. Comprehensive characterization of RAS mutations in colon and rectal cancers in old and young patients. Nat. Commun. 10, 3722 (2019).

102. Xiu, C. et al. Novel benzopyran derivatives and their therapeutic applications: a patent review (2009-2016). Expert Opin. Ther. Pat. 27, 1031-1045 (2017).

103. Zhang, L. et al. IMCA induces ferroptosis mediated by SLC7A11 through the AMPK/mTOR pathway in colorectal cancer. Oxid. Med. Cell Longev. 2020 1675613 (2020).

104. Izumi, D. et al. Colorectal cancer stem cells acquire chemoresistance through the upregulation of F-BoxMD repeat-containing protein 7 and the consequent degradation of c-Myc. Stem Cells 35, 2027-2036 (2017).

105. $\mathrm{Xu}, \mathrm{X}$. et al. Targeting SLC7A11 specifically suppresses the progression of colorectal cancer stem cells via inducing ferroptosis. Eur. J. Pharm. Sci. 152, 105450 (2020).

106. Park, S., Oh, J., Kim, M. \& Jin, E. J. Bromelain effectively suppresses Kras-mutant colorectal cancer by stimulating ferroptosis. Anim. Cells Syst. (Seoul.) 22 334-340 (2018).

107. Angius, A. et al. Integrated analysis of miRNA and mRNA endorses a twenty mirnas signature for colorectal carcinoma. Int. J. Mol. Sci. 20, 4067 (2019).

108. Hong, S. H. et al. Molecular crosstalk between ferroptosis and apoptosis: emerging role of ER stress-induced p53-independent PUMA expression. Oncotarget 8, 115164-115178 (2017).

109. Lee, Y. S. et al. Ferroptosis-inducing agents enhance TRAlL-induced apoptosis through upregulation of death receptor 5. J. Cell Biochem. 120, 928-939 (2019).

110. Hasan, M., Reddy, S. M. \& Das, N. K. Ferritinophagy is not required for colon cancer cell growth. Cell Biol. Int. 44, 2307-2314 (2020).

111. Li, Y. et al. H(2) S-scavenged and activated iron oxide-hydroxide nanospindles for MRI-guided photothermal therapy and ferroptosis in colon cancer. Small 16, e2001356 (2020)

112. Lu, D. et al. ACADSB regulates ferroptosis and affects the migration, invasion and proliferation of colorectal cancer cells. Cell Biol. Int. 44, 2334-2343 (2020).

113. Ousingsawat, J., Schreiber, R. \& Kunzelmann, K. TMEM16F/Anoctamin 6 in ferroptotic cell death. Cancers (Basel) 11, 625 (2019). 